\title{
Production du fourrage de Mucuna pruriens pour l'alimentation des animaux et sa marge brute en zone cotonnière du Mali
}

\author{
Sidi Oumar TRAORE ${ }^{1 *}$, Alassane BA ${ }^{1}$, Doubangolo COULIBALY ${ }^{2}$, \\ Abdoul Kader KONE ${ }^{1}$, Bouréma KONE ${ }^{3}$ et Nangboro Ferdinand DEMBELE ${ }^{1}$ \\ ${ }^{I}$ Institut d'Economie Rurale (IER), Centre Régional de Recherche Agronomique (CRRA) de Sikasso, \\ Délégation Programme Bovin/Camelin, BP : 16, Sikasso, Mali. \\ ${ }^{2}$ Institut d'Economie Rurale (IER), Centre Régional de Recherche Agronomique (CRRA) de Sotuba, \\ Programme Bovin/Camelin, BP : 262, Bamako, Mali. \\ ${ }^{3}$ Institut d'Economie Rurale (IER), Centre Régional de Recherche Agronomique (CRRA) de Sotuba, \\ Programme ECOnomie des FILières (ECOFIL), BP : 262, Bamako, Mali. \\ *Auteur correspondant ; E-mail : traoresidioumar@gmail.com; Tél. : (00223) 76938949 / (00223) 66966910 \\ Received: 09-10-2020 $\quad$ Accepted: 16-02-2021 $\quad$ Published: 28-02-2021

\section{RESUME} \\ En zone cotonnière du Mali, la situation d'affouragement des animaux est largement déficitaire. Pour \\ améliorer la disponibilité des ressources fourragères, la recherche en partenariat avec les producteurs a introduit \\ en milieu paysan la culture de Mucuna pruriens. Cette étude vise à évaluer les performances de M. pruriens dans \\ la production du fourrage pour les animaux. Les essais ont été conduits par 25 producteurs répartis dans 5 sites \\ durant les campagnes agricoles 2015-2016, 2016-2017 et 2017-2018. Les essais étaient divisés en deux parcelles \\ tests : culture pure de $M$. pruriens et association $M$. pruriens/maïs. La production moyenne en biomasse de $M$. \\ pruriens pur sur les 3 campagnes agricoles a été de $4363 \pm 1491 \mathrm{~kg}$ MS/ha. Cette production couvre les besoins \\ en matière sèche de 7,76 $\pm 2,65$ UBT durant 90 jours. Pour l'association, la production de biomasse a été de 5 \\ 449 $11766 \mathrm{~kg} \mathrm{MS/ha,} \mathrm{elle} \mathrm{couvre} \mathrm{les} \mathrm{besoins} \mathrm{en} \mathrm{matière} \mathrm{sèche} \mathrm{de} \mathrm{9,69 \pm 3,14} \mathrm{UBT} \mathrm{pendant} 90$ jours. La marge \\ brute de la culture pure de $M$. pruriens est de 786 060 \pm 298140 FCFA/ha. Pour l'association, elle est de 850

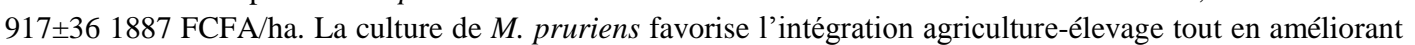 \\ l'alimentation des animaux et les revenus des producteurs.
}

(C) 2021 International Formulae Group. All rights reserved.

Mots clés: Exploitation agricole, date de semis, Cultures fourragères, $M$. pruriens, zone cotonnière.

\section{Production of Mucuna pruriens fodder for animal feed and its gross margin in the cotton-growing zone of Mali}

\begin{abstract}
In Mali's cotton-growing zone, there is a large deficit in animal feed. To improve the availability of fodder resources, research in partnership with producers has introduced the cultivation of Mucuna pruriens into the farming environment. This study aims ed at evaluat'hg the performance of $M$. pruriens in the production of fodder for animals. The trials were conducted by 25 producers in 5 sites during the 2015-2016, 2016-2017 and
\end{abstract}


2017-2018 crop years. The trials were divided into two test plots: pure culture of $M$. pruriens and $M$. pruriens/corn combination. The average biomass production of pure $M$. pruriens over the 3 cropping seasons was $4363 \pm 1,491 \mathrm{~kg}$ MS/ha. This production covers the dry matter requirement of $7.76 \pm 2.65$ Btu for 90 days. For the association, the biomass production was $5449 \pm 1,766 \mathrm{~kg} \mathrm{DM} / \mathrm{ha}$, covering the dry matter requirement of $9.69 \pm 3.14$ Btu for 90 days. The gross margin of the pure culture of M. pruriens is $786060 \pm 298140 \mathrm{FCFA} / \mathrm{ha}$. For the association, it is $850917 \pm 361887 \mathrm{FCFA} / \mathrm{ha}$. The cultivation of $M$. pruriens promotes the integration of agriculture and livestock while improving animal nutrition and the income of producers.

(C) 2021 International Formulae Group. All rights reserved.

Keywords: Farm, sowing date, fodder crops, M. pruriens, cotton area.

\section{INTRODUCTION}

En Afrique subsaharienne, l'alimentation des animaux repose encore entièrement sur l'exploitation des jachères, des savanes et autres formations végétales (Coulibaly et al., 2009 ; Djenontin et al., 2009). Le pâturage naturel est dégradé aujourd'hui par des facteurs non seulement climatiques mais aussi anthropiques (Coulibaly et al., 2017). Dans les zones cotonnières de l'Afrique de l'Ouest, les exploitants agricoles qui sont des agro éleveurs valorisent bien la fumure organique, utilisent la traction animale mais malheureusement ne disposent pas suffisamment de fourrages pour l'alimentation des animaux (Koutou et al., 2016).

$\mathrm{Au} \quad$ Mali, l'accroissement démographique (INSTAT, 2016), l'adoption progressive de la traction attelée et l'orientation commerciale de certaines cultures (coton) ont profondément modifié les conditions foncières (Sanogo et al., 2010). Cette modification se traduit par l'augmentation des superficies cultivées au détriment des zones de pâturage. Ce type d'utilisation déséquilibrée de l'espace et de ses ressources se traduit par une dégradation progressive en qualité ainsi qu'en quantité des ressources fourragères naturelles (Blanchard, 2010).

En zone cotonnière du Mali, cette dégradation des ressources fourragères est perceptible et accentuée sous l'influence des aléas climatiques (Coulibaly et al., 2017; Sissoko et al., 2020). En conséquence, la situation d'affouragement des animaux est largement déficitaire tandis que l'effectif du cheptel ne cesse de s'accroître (Coulibaly, 2008). En outre, des études menées dans la zone cotonnière ont confirmé que les parcours naturels, les résidus de culture stockés, les aliments concentrés achetés ne permettent plus d'accroître la productivité des animaux en termes de reproduction, de production de lait, de la viande et de la fumure organique ainsi que la fourniture d'énergie (Ba et al., 2011 ; Coulibaly et al., 2017).

La production du fourrage en quantité et en qualité devient de plus en plus une nécessité pour améliorer la productivité des animaux (Klein et al., 2014). Ainsi, la culture des légumineuses fourragères permet d'obtenir une quantité importante de fourrage de qualité en raison de leur teneur élevée en matière azotée et en énergie (Coulibaly et al., 2012 ; Kiema et al., 2012). Ainsi, pour accroître les ressources alimentaires des animaux et améliorer les revenus des agro-éleveurs, la recherche agricole a introduit la culture de $M$. pruriens en milieu paysan au Mali pendant les campagnes agricoles 2015-2016, 2016-2017 et 2017-2018.

L'espèce $M$. pruriens est une légumineuse fourragère qui a de bonnes performances biologiques grâce à une production rapide et importante de biomasses aériennes (Haro et al., 2020). La biomasse ainsi que les graines sont composées des éléments nutritifs pour les animaux et elles peuvent procurer des intérêts économiques pour les petits exploitants agricoles (Hauser et Nolte, 2002 ; Coulibaly et al., 2012). L'espèce a été également retenue à cause de la disponibilité de ses semences dans la région.

Cette étude s'intéresse aux performances de la culture de M. pruriens dans la production du fourrage tout en déterminant les périodes optimales de semis dans la zone cotonnière du Mali. Nos travaux cherchent aussi à déterminer les performances 
économiques et la charge animale autorisée par unité de superficie de $M$. pruriens en culture pure et en culture associée au maïs pour son appropriation par les petits exploitants agricoles.

\section{MATERIEL ET METHODES Zone d'étude}

L'étude a concerné 5 villages à travers les régions agricoles de la zone cotonnière du Mali (Figure 1). Il s'agit : Kafara, Benguéné, Ziguéna, Kokélé et Nafégué. Le choix des villages a été fait sur la base du zonage agroécologique suivant certains critères dont la diversité des systèmes de production agricole, l'accessibilité et l'absence d'autres projets de recherche-développement dans les villages (Soumaré et al., 2008).

Dans ces villages, les activités agricoles sont basées sur la culture du coton, le maïs, le sorgho, le mil, le riz, l'arachide, patate douce, le niébé etc. L'élevage est pratiqué par plus de 90\% des habitants de la zone. Cet élevage joue un grand rôle dans le transport des produits agricoles et les opérations culturales (labour, sarclage, semis etc.).

La pluviométrie a été variable entre les villages d'étude au cours des trois campagnes agricoles. La moyenne pluviométrique sur les trois campagnes agricoles a été de $1027 \mathrm{~mm}$ à Nafégué, $1113 \mathrm{~mm}$ à Ziguéna, $1182 \mathrm{~mm}$ à Kokélé, $951 \mathrm{~mm}$ à Kafara et $876 \mathrm{~mm}$ à Benguéné.

\section{Matériel végétal}

Le matériel végétal était : maïs (Zea mays L.) var. IITA-CYMT/ Mali IER, 1985, nom commun Sotubaka et Mucuna pruriens (L.) DC var. utilis (Wall. ex Wight) Baker ex Burck.

\section{Dispositif expérimental}

Le dispositif utilisé a été un bloc dispersé avec 2 parcelles tests :

- Test 1 : Culture pure de M. pruriens sur une superficie de 0,25 ha;

- Test 2 : Culture associée de $M$. pruriens au maïs sur une superficie de 0,25 ha.
La parcelle de chaque producteur représente une répétition. Les tests ont été conduits chez 5 producteurs volontaires par village sur des parcelles différentes (rotation légumineuses-céréales) pendant 3 campagnes agricoles.

\section{Variables collectées pour les cultures}

Les productions moyennes en biomasse de chaque village ont été calculées en fonction des campagnes agricoles. Pour déterminer l'effet des périodes de semis sur les rendements des cultures, les dates de semis ont été regroupées en 3 décades du mois (tous les 10 jours). La première décade $=\mathrm{D} 1$, la deuxième décade $=\mathrm{D} 2$ et la troisième décade $=\mathrm{D} 3$.

\section{Itinéraires techniques des expérimentations}

Le semis de M. pruriens en culture pure a été fait aux écartements de $80 \mathrm{~cm}$ entre les lignes et $80 \mathrm{~cm}$ entre les poquets à raison de deux graines par poquet (soit $30 \mathrm{~kg} / \mathrm{ha}$ de semences). La fertilisation minérale, le DAP (18 N-6P205-0) a été apporté entre le $10^{\text {ième }}$ et le $15^{\text {ième }}$ après la levée à la dose de $65 \mathrm{~kg} / \mathrm{ha}$.

Pour l'association, le maïs a été semé aux écartements de $80 \mathrm{~cm} \mathrm{x} 40 \mathrm{~cm}$ à raison de deux à quatre graines par poquet. Le démariage a été fait à 2 plants/poquet au $15^{\text {ième }}$ Jour Après le Semis (JAS). Le semis de M. pruriens a été effectué 25 jours après le semis du maïs et sur la même ligne que le maïs aux écartements de $80 \mathrm{~cm}$ entre les poquets, à raison de deux graines par poquet. Le maïs a reçu $100 \mathrm{~kg} / \mathrm{ha}$ de NPK (15 N-15 P-15 K) au semis et 150 $\mathrm{kg} / \mathrm{ha} \mathrm{d}$ drée $(46 \% \mathrm{~N})$ au $45^{\text {ième }} \mathrm{JAS}$.

Les parcelles ont été sarclées 2 fois par campagne. La fauche du fourrage de $M$. pruriens a été effectuée à $50 \%$ de floraison (entre 8 et 12 semaines après le semis). En association, les tiges de maïs et les fanes de $M$. pruriens ont été fauchées ensemble.

\section{Estimation de la biomasse}

La méthode d'estimation de la biomasse consistait en une délimitation de 3 carrés de 5 $\mathrm{m}$ x $5 \mathrm{~m}$ dans chaque parcelle test suivant la diagonale. Chaque carré a été récolté et pesé individuellement. Un échantillon frais de $2 \mathrm{~kg}$ pour chaque culture a été prélevé et séché à 
l'air ambiant pendant quelques jours pour déterminer le poids sec de chaque échantillon.

\section{Caractéristiques des exploitations agricoles (EA)}

Une enquête socio-économique a été effectuée pour caractériser les EA des villages d'étude. L'analyse a porté sur les variables suivantes : l'âge du chef d'exploitation, la taille de l'exploitation, les superficies cultivées, l'effectif du cheptel et le nombre de charrue.

\section{Calcul de la charge animale}

Le calcul de la charge animale permise par la biomasse produite a été fait à partir des taux de conversion établis par Boudet \& Rivière (1968). Un animal de $250 \mathrm{~kg}$ de poids vif correspond en une Unité de Bétail Tropical (UBT) et sa consommation journalière est évaluée à $6,25 \mathrm{~kg}$ de MS (Boudet et Rivière, 1968). Les besoins de l'UBT sont rapportés au $\mathrm{kg}$ de matières sèches obtenues à l'hectare sur la moyenne des 3 campagnes agricoles pour estimer le nombre d'UBT à nourrir durant 90 jours. Les 90 jours correspondent aux 3 mois de la saison sèche, période où la pénurie d'aliment est importante dans la zone d'étude.

\section{Calcul des variables économiques}

L'analyse financière a concerné la marge brute (MB). Elle est la différence entre la valeur brute (VB) et les charges variables totales (CVT). La formule est donnée comme suit :

$$
\mathrm{MB}(\pi)=\sum \mathrm{VB}-\sum \mathrm{CVT}=\sum \mathrm{PQ}-\sum \mathrm{PX}
$$

$\mathrm{Q}$ correspond à la quantité (fourrage et graines), $\mathrm{X}$ correspond aux inputs et $\mathrm{P}$ correspond aux prix.

Pour estimer la VB, la production du maïs graine et de biomasse à l'hectare des cultures a été valorisée en chiffre monétaire (100 FCFA/kg de maïs graine, $200 \mathrm{FCFA} / \mathrm{kg}$ de fanes de M. pruriens pur et 150 FCFA pour le mélange du fourrage de maïs et de $M$. pruriens). Ces prix ont été déterminés en se référant aux prix moyens des fourrages de niébé et d'arachide dans les marchés locaux après une enquête. Les CVT ont été obtenus en faisant la somme des coûts d'intrants (semences, herbicide, engrais) et les coûts des opérations culturales (labour, semis, sarclage, récolte, transport). Le coût de labour est estimé à $20000 \mathrm{FCFA} / \mathrm{ha}$. Pour les autres opérations, les coûts sont estimés en fonction du tarif moyen journalier dans la zone d'étude (750 FCFA/homme/jour).

\section{Analyse et traitement des données}

Les analyses ont porté sur la statistique descriptive avec les logiciels $\mathrm{R}$ et Excel. L'analyse de variance suivie de la comparaison des moyennes selon le test de Newman et Keuls a été utilisée pour le traitement des données à l'aide du logiciel R-3.5.1. Les effets des tests ont été considérés comme significatifs au seuil de $5 \%$ et de $1 \%$.

\section{RESULTATS}

\section{Caractéristiques des exploitations agricoles de la zone d'étude}

Les Exploitants Agricoles (EA) dans la zone sont des agro-éleveurs qui combinent les activités de l'agriculture et de l'élevage (Tableau 1). Le cheptel est composé principalement des bovins, caprins, ovins et asins. Le nombre moyen d'UBT par EA est très variable entre les villages d'étude. Il est de moins de 10 UBT dans les villages de Kafara et Kokélé à plus de 20 UBT dans les villages de Ziguéna et Nafégué. Le système cotoncéréales (maïs, sorgho, mil) est le plus important. Les EA qui ont les plus grands nombres d'UBT cultivent plus de coton. Pour les légumineuses (arachide et niébé), les superficies cultivées sont inférieures à celles des autres cultures dans tous les villages.

\section{Production de biomasse de la culture pure} de $M$. pruriens et associé au maïs par campagne agricole dans les sites d'étude

L'analyse de variance a montré des différences significatives entre les villages pour la production en biomasse de la culture pure de $M$. pruriens au cours des 3 campagnes agricoles. Elle a montré également des différences significatives entre les sites d'étude pour ce qui concerne la production de biomasse obtenue dans l'association (Tableau 2). La meilleure production (5826 kg MS/ha) de biomasse de $M$. pruriens en culture pure a été 
obtenue en 2016-2017 dans le village de Kokélé. Celle de la faible production $(2347 \mathrm{~kg}$ $\mathrm{MS} / \mathrm{ha}$ ) a été obtenue à Benguéné en 20152016. Pour la production dans l'association de la culture de $M$. pruriens au maïs, Kokélé a également produit la meilleure production (7169 kg MS/ha) en 2016-2017. La faible production de biomasse (4050 $\mathrm{kg} \mathrm{MS} / \mathrm{ha}$ ) a été observée dans le village de Nafégué en 2017 2018.

Dans l'ensemble des villages d'étude, les productions moyennes de $M$. pruriens en culture pure $(4877 \pm 1349)$ et en association avec le maïs $(5870 \pm 1787)$ ont été meilleures lors de la deuxième campagne agricole (20162017). Aussi, les productions moyennes obtenues dans la culture pure ainsi qu'en association lors de la dernière campagne agricole (2017-2018) sont légèrement inférieures à celles obtenues pendant la première campagne agricole (2015-2016).

\section{Production de biomasse de la culture de $M$.} pruriens pur et associé au maïs selon les dates de semis

Au cours des 3 campagnes agricoles, les parcelles ont été implantées de la deuxième décade du mois de juin à la première décade du mois de septembre (Figure 2). L'analyse de variance a montré des différences significatives $(p<0,001)$ entre les productions moyennes de biomasse de $M$. pruriens en pur et en association avec le maïs pour les différentes périodes de semis dans sites d'étude. La meilleure production de $M$. pruriens en culture pure a été observée dans la deuxième décade du mois de juin $\mathrm{La}$ production baisse considérablement à partir de la première décade du mois d'août (3817 kg MS/ha). Pour l'association de la culture de M. pruriens au maïs les meilleures productions ont été obtenues sur les parcelles semées dans la deuxième $(5126 \mathrm{~kg} \mathrm{MS} / \mathrm{ha})$ et la troisième décade (5026 kg MS/ha) du mois de juillet (Figure 2). La production baisse considérablement de la première décade du mois d'août (4293 kg MS/ha) vers la troisième décade (3852 kg MS/ha). Les parcelles semées dans la première décade du mois de septembre ont donné la plus faible production en biomasse (2290 kg MS/ha).

Estimation de la charge animale à partir de la biomasse de $M$. pruriens pur et associé au maïs dans les villages d'études

L'analyse de variance a montré des différences significatives $(p<0,001)$ entre les villages pour le nombre moyen d'UBT/ha à affourager pendant 90 jours (Tableau 3). Dans l'ensemble des sites, la production moyenne à l'hectare en biomasse de $M$. pruriens pur sur les trois campagnes agricoles $(4363 \pm 1491 \mathrm{~kg}$ $\mathrm{MS} / \mathrm{ha}$ ) est suffisante pour couvrir les besoins en matières sèche de 7,76 $\pm 2,65 \mathrm{UBT} /$ ha durant 90 jours. Dans le cas de l'association, la production moyenne en biomasse a été de $5449 \pm 1766 \mathrm{~kg} \mathrm{MS} / \mathrm{ha}$. Cette production couvre les besoins en matière sèche de $9,69 \pm 3,14$ UBT/ha pendant 90 jours.

Rentabilité économique de la culture de $M$. pruriens pur et associé au maïs dans les sites d'étude

L'analyse de variance a montré des différences hautement significatives $(p<0,001)$ entre les différents sites d'étude pour les marges brutes obtenues de la culture pure de $M$. pruriens et de l'association du maïs au $M$. pruriens (Tableau 4). Les technologies sont financièrement rentables en termes de marge brute pour les producteurs. Pour la culture de M. pruriens associé au maïs dans l'ensemble des sites d'étude, la marge brute a été de $850917 \pm 361887$ FCFA/ha sur les trois campagnes agricoles. En culture pure, la marge brute a été de $786060 \pm 298140$ FCFA/ha. Dans les villages de Nafégué, Ziguéna et Kokélé la marge brute obtenue dans l'association est supérieure à celle obtenue en culture pure de $M$. pruriens. Or dans les villages de Benguéné et Kafara la marge brute de la culture pure du $M$. pruriens est supérieure à celle obtenue dans l'assation. 
Tableau 1 : Caractéristiques des exploitants agricoles.

\begin{tabular}{|c|c|c|c|c|c|c|c|c|c|c|}
\hline \multirow[t]{2}{*}{ Village } & \multirow{2}{*}{$\begin{array}{c}\text { Age } \\
\text { CE (an) }\end{array}$} & \multirow{2}{*}{$\begin{array}{l}\text { Nombre de } \\
\text { Personnes }\end{array}$} & \multicolumn{4}{|c|}{ Superficie (ha) } & \multicolumn{2}{|c|}{ Légumineuses (ha) } & \multirow{2}{*}{$\begin{array}{l}\text { Cheptel } \\
\text { (UBT) }\end{array}$} & \multirow{2}{*}{$\begin{array}{l}\text { Charrue } \\
\text { (nombre) }\end{array}$} \\
\hline & & & Maïs & Sorgho & Mil & Coton & Arachide & Niébé & & \\
\hline Nafégué & $53 \pm 13$ & $18 \pm 13$ & $3,90 \pm 2,39$ & $0,76 \pm 0,31$ & $1,04 \pm 0,48$ & $3,87 \pm 2,28$ & $0,94 \pm 0,57$ & $0,52 \pm 0,37$ & $22,26 \pm 11,84$ & $1,58 \pm 0,85$ \\
\hline Ziguéna & $55 \pm 14$ & $22 \pm 16$ & $4,33 \pm 2,89$ & $2,05 \pm 1,68$ & $1,08 \pm 0,59$ & $7,36 \pm 6,13$ & $0,33 \pm 0,18$ & $0,31 \pm 0,13$ & $26,10 \pm 12,55$ & $1,83 \pm 1,16$ \\
\hline Benguéné & $54 \pm 15$ & $22 \pm 21$ & $1,51 \pm 1,24$ & $2,04 \pm 1,71$ & $5,07 \pm 3,43$ & $2,35 \pm 2,02$ & $1,08 \pm 1,17$ & $0,74 \pm 0,69$ & $11,58 \pm 5,92$ & $1,94 \pm 1,33$ \\
\hline Kafara & $63 \pm 14$ & $20 \pm 11$ & $1,66 \pm 0,60$ & $3,51 \pm 2,01$ & $1,63 \pm 1,59$ & $1,73 \pm 1,15$ & $1,35 \pm 0,94$ & $0,60 \pm 0,34$ & $7,41 \pm 4,01$ & $1,07 \pm 0,54$ \\
\hline Kokélé & $54 \pm 16$ & $17 \pm 12$ & $1,54 \pm 1,13$ & $1,68 \pm 1,12$ & $1,38 \pm 1,18$ & $1,31 \pm 0,93$ & $0,60 \pm 0,45$ & $0,52 \pm 0,40$ & $5,76 \pm 4,12$ & $0,93 \pm 0,72$ \\
\hline Probabilité & $0,04 * *$ & $0,01 * *$ & $0,001 * * *$ & $0,001 * * *$ & $0,001 * * *$ & $0,001 * * *$ & $0,001 * * *$ & $0,004 * * *$ & $0,001 * * *$ & $0,02 * *$ \\
\hline
\end{tabular}

Légende : CE : Chef d'Exploitation ; ha : Hectare ; \pm : Ecart-type ; *** : Significatif à $1 \%$; ** : Significatif à $5 \%$; UBT : Unité de Bétail Tropical

Tableau 2 : Production de biomasse de M.pruriens en pur et en association avec le maïs par campagne agricole.

\begin{tabular}{|c|c|c|c|c|c|c|}
\hline \multirow[t]{2}{*}{ Village } & \multicolumn{4}{|c|}{ M. pruriens pur (kg MS/ha) } & \multicolumn{2}{|c|}{ Maïs/M. pruriens (kg MS/ha) } \\
\hline & Campagne 2015-2016 & Campagne 2016-2017 & Campagne 2017-2018 & Campagne 2015-2016 & Campagne 2016-2017 & Campagne 2017-2018 \\
\hline \multirow[t]{2}{*}{ Nafégué } & 4537 & 5572 & 3470 & 5968 & 6362 & 4050 \\
\hline & \pm 1768 & \pm 976 & \pm 962 & \pm 1488 & \pm 876 & \pm 705 \\
\hline \multirow[t]{2}{*}{ Ziguéna } & 4508 & 4445 & 4356 & 7020 & 6190 & 6275 \\
\hline & \pm 1204 & \pm 1398 & \pm 871 & \pm 585 & \pm 1250 & \pm 1552 \\
\hline \multirow[t]{2}{*}{ Benguéné } & 2347 & 3170 & 4335 & 5300 & 5445 & 5124 \\
\hline & \pm 1635 & \pm 591 & \pm 1539 & \pm 1320 & \pm 2343 & \pm 1829 \\
\hline \multirow[t]{2}{*}{ Kafara } & 4745 & 5372 & 3781 & 3612 & 4184 & 5127 \\
\hline & \pm 1730 & \pm 964 & \pm 1467 & \pm 1576 & \pm 1436 & \pm 1120 \\
\hline \multirow[t]{2}{*}{ Kokélé } & 5817 & 5826 & 3157 & 6305 & 7169 & 4991 \\
\hline & \pm 925 & \pm 907 & \pm 196 & \pm 888 & \pm 1718 & \pm 2894 \\
\hline \multirow[t]{2}{*}{ Moyenne } & 4391 & 4877 & 3820 & 5726 & 5870 & 5113 \\
\hline & \pm 1782 & \pm 1349 & \pm 1128 & \pm 1717 & \pm 1787 & \pm 1779 \\
\hline Probabilité & $<0,001 * * *$ & $<0,001 * * *$ & $0,04 * *$ & $<0,001 * * *$ & $<0,001 * * *$ & $0,008 * *$ \\
\hline
\end{tabular}

Légende : $\pm=$ Ecart type ; $* * *=$ Significatif à $1 \% ; * *=$ Significatif à $5 \%$ 


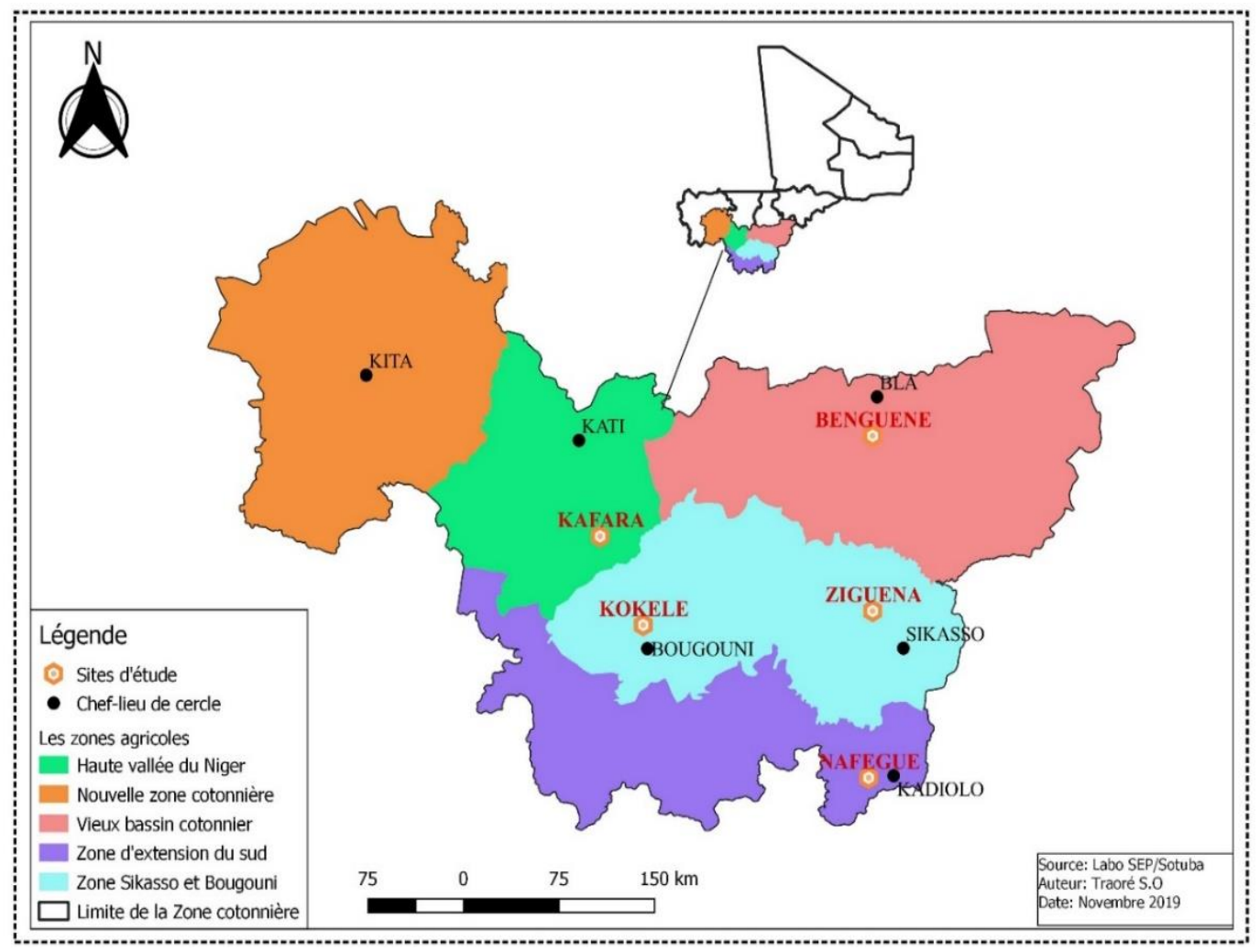

Figure 1: Localisation de la zone d'étude.

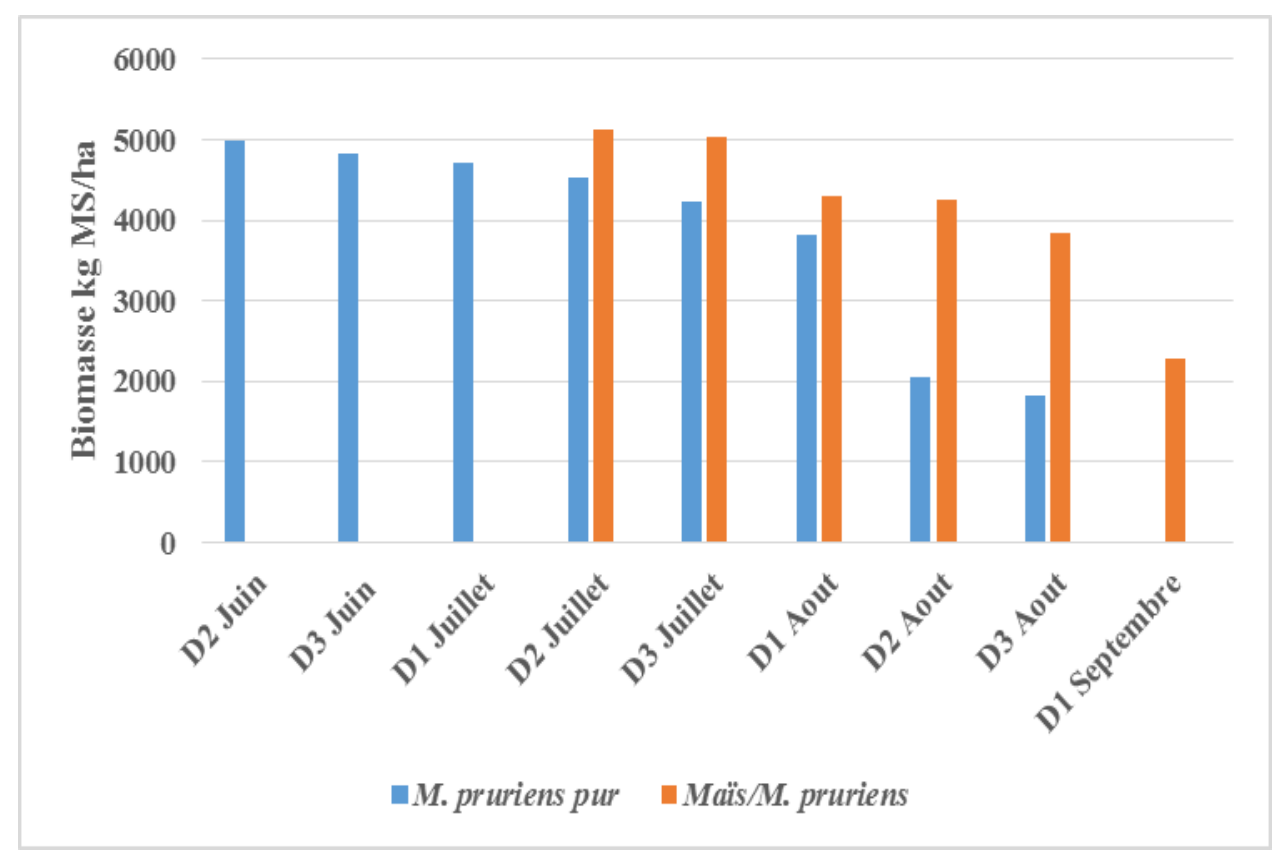

Figure 2 : Production de biomasse de la culture pure de Mucuna et associé au maïs selon les dates de semis. 
Tableau 3 : Estimation de la charge animale à partir de la production de biomasse de M. pruriens pur et associé au maïs durant 90 jours.

\begin{tabular}{lcccc}
\hline \multirow{2}{*}{ Village } & \multicolumn{2}{c}{ M. pruriens pur } & \multicolumn{2}{c}{ Maïs/M. pruriens } \\
\cline { 2 - 5 } & $\begin{array}{c}\text { Production biomasse } \\
\text { (MS kg/ha) }\end{array}$ & $\begin{array}{c}\text { Charge animale } \\
\text { (UBT/ha) }\end{array}$ & $\begin{array}{c}\text { Production biomasse } \\
\text { (MS kg/ha) }\end{array}$ & $\begin{array}{c}\text { Charge animale } \\
\text { (UBT/ha) }\end{array}$ \\
\hline Nafégué & $4526 \pm 1489$ & $8,05 \pm 2,65$ & $5460 \pm 1445$ & $9,71 \pm 2,57$ \\
\hline Ziguéna & $4436 \pm 1092$ & $7,89 \pm 1,94$ & $6549 \pm 1063$ & $11,64 \pm 1,89$ \\
\hline Benguéné & $3284 \pm 1501$ & $5,84 \pm 2,67$ & $4771 \pm 1777$ & $8,48 \pm 3,16$ \\
\hline Kafara & $4633 \pm 1481$ & $8,24 \pm 2,63$ & $4308 \pm 1441$ & $7,66 \pm 2,56$ \\
\hline Kokélé & $4933 \pm 1477$ & $8,77 \pm 2,63$ & $6155 \pm 2078$ & $10,94 \pm 3,69$ \\
\hline Moyenne & $4363 \pm 1491$ & $7,76 \pm 2,65$ & $5449 \pm 1766$ & $9,69 \pm 3,14$ \\
\hline Probabilité & $<0,001 * * *$ & $<0,001 * * *$ & $<0,001 * * *$ & $<0,001 * * *$ \\
\hline
\end{tabular}

Légende : CE : *** : Significatif à $1 \%$; UBT : Unité de Bétail Tropical ; MS : Matières Sèches ; \pm : Ecart-type

Tableau 4 : Marge brute de la culture de M. pruriens pur et associé au maïs dans les sites d'étude durant les trois campagnes agricoles.

\section{Marge brute en FCFA/ha}

\begin{tabular}{lcc}
\cline { 2 - 3 } Village & M. pruriens & Maïs/M. pruriens \\
\hline Nafégué & $818803 \pm 297834$ & $930944 \pm 247493$ \\
\hline Ziguéna & $800790 \pm 218498$ & $1008454 \pm 176689$ \\
\hline Benguéné & $570394 \pm 300179$ & $555859 \pm 544407$ \\
\hline Kafara & $840083 \pm 296220$ & $758787 \pm 233289$ \\
\hline Kokélé & $900230 \pm 295337$ & $1000539 \pm 298571$ \\
\hline Moyenne & $786060 \pm 298140$ & $850917 \pm 361887$ \\
\hline Probabilité & $<0,001 * * *$ & $<0,001 * * *$
\end{tabular}

Légende : *** : Significatif à $1 \% ; \pm$ : Ecart-type 


\section{DISCUSSION}

\section{Facteurs influents la production de biomasse de la culture de $M$. pruriens}

La production de la biomasse de $M$. pruriens est influencée très généralement par le niveau de technicité des producteurs (Coulibaly et al., 2017), de la pluviométrie et les périodes de semis. La production de biomasse de $M$. pruriens la plus élevée en culture pure et en association parmi les trois campagnes agricoles a été obtenue à Kokélé dans la filiale Sud où la pluviométrie a été meilleure avec une hauteur de $1182 \mathrm{~mm}$. La plus faible production de biomasse a été obtenue dans le village de Benguéné où la pluviométrie a été inferieure par rapport aux autres sites d'étude. Lors campagne agricole 2017-2018, dernière campagne, la production moyenne de la culture pure (3 $820 \pm 1128 \mathrm{~kg}$ MS/ha) a été inférieure aux moyennes des autres. Ce résultat est inférieur à un rendement de $5900 \mathrm{~kg}$ obtenus par Hauser and Nolte (2002) et nettement supérieur à celui obtenu (1,43 t/ha) par Koutou et al. (2009). Dans l'association la production a été meilleure en 2017 avec une moyenne de $5870 \pm 1787 \mathrm{~kg}$ MS/ha. Ce résultat est inférieur à ceux trouvés par Carsky (2003) avec une moyenne de 11000 $\mathrm{kg} \mathrm{MS/ha.} \mathrm{Il} \mathrm{est} \mathrm{supérieur} \mathrm{à} \mathrm{un} \mathrm{rendement} \mathrm{de} 4$ $200 \pm 600 \mathrm{~kg} \mathrm{MS} / \mathrm{ha}$ trouvé par Pamba et al. (2018) sous une pluviométrie moyenne de 1 200 à $1500 \mathrm{~mm}$ et supérieur également à une moyenne de $4113 \mathrm{~kg}$ MS/ha trouvé par Coulibaly et al. (2017).

Pour les périodes de semis au cours des 3 campagnes agricoles, la meilleure production de biomasse de $M$. pruriens en pur a été obtenue dans la deuxième décade du mois de juin (Figure 2). La production baisse considérablement à partir de la première décade du mois d'août. Cette situation s'explique par le fait que les plantes semées dès le mois de juin ou juillet sous les conditions climatiques de la zone ont beaucoup de chance de recevoir plus d'eau pour leur croissance et développement végétatif. En plus, pendant la première décade du mois d'août, les pluies ont été très abondantes dans la zone, ce qui peut exposer les sols à la saturation d'eau. Il y aura donc une mauvaise aération des sols qui influencerait négativement la levée et le développement végétatif des plantes (Koutou et al., 2009). Par contre, avant la mi-juillet, les sols seraient bien humidifiés et bien aérés, donc favorable à une bonne levée et un bon enracinement des plantes. La culture aurait eu également le temps de boucler son cycle de développement végétatif avant l'arrêt des pluies. Diatta et al. 2020 ont obtenu des rendements en fourrage de Mucuna très faibles due à l'installation tardive des plantes couplée aux insuffisances pluviométriques. Ces derniers ont obtenu une moyenne de 1,16 à 1,50 t MS/ ha qui sont proches à nos résultats des parcelles installées tardivement.

Dans le cas de l'association si le semis de M. pruriens est effectué sous le maïs très tôt, il peut l'étouffer rapidement car c'est une espèce rampante dont sa croissance végétative est très rapide. De même, quand on fait le semis de $M$. pruriens sous le maïs trop tard aussi, la plante $(M$. pruriens) ne pourra pas se développer normalement sous l'effet de la chaleur ou l'ombrage de son tuteur (maïs) qui engendre une diminution considérable des rendements de la biomasse (Coulibaly, 2017).

\section{Amélioration de l'alimentation des animaux}

Le nombre d'UBT à nourrir durant 90 jours à partir du fourrage de $\mathrm{M}$. pruriens en pur et en association avec le mais sur les trois campagnes agricoles varie d'un village à un autre. Cela s'explique par la variation de la production de biomasse en fonction des campagnes dans les différents villages d'étude. Le nombre moyen d'UBT à affourager avec la biomasse produite de $M$. pruriens associé au maïs est supérieur à celui obtenu dans la culture pure de $M$. pruriens dans chaque village d'étude. Les fourrages obtenus dans l'association ont été supérieurs à ceux obtenus dans la culture pure. Dans l'ensemble des sites, le nombre moyen d'UBT à nourrir avec la biomasse de $M$. pruriens en pur est de 7,76 UBT/ha. Pour le cas de l'association, il s'élève à 9,69 UBT/ha à nourrir pendant 90 jours. Ce résultat est similaire à celui trouvé par Coulibaly et al. (2017) avec une moyenne de 9,13 UBT/ha à nourrir pendant 90 jours. 
Dans les villages de Kafara et de kokélé, la biomasse produite à l'hectare dans l'association de M. pruriens au maïs couvre les besoins en matières sèches durant 90 jours des nombres moyens d'UBT par exploitation que disposent ces villages. Le nombre moyen d'UBT par exploitation est faible dans ces villages par rapport aux autres villages d'étude (Tableau 1). Par contre, dans les villages de Ziguéna, Nafégué et Benguéné la biomasse produite à l'hectare ne couvre pas les besoins en matières sèches des nombres moyens d'UBT par exploitation pendant les 90 jours de la période sèche. Compte tenu des contraintes liées à l'extension des superficies des cultures, ces villages peuvent augmenter les superficies dans l'association.

\section{Sources de revenus des producteurs de la zone}

L'adoption d'une culture dépend généralement de sa performance économique. L'étude révèle que la culture de $M$. pruriens est rentable économiquement pour les producteurs. Cependant, la rentabilité économique en termes de marge brute est beaucoup influencée par les dépenses effectuées aux opérations de récolte (fauche, séchage et conservation du fourrage).

Dans l'ensemble des villages d'étude, la marge brute tirée d'un hectare de la culture pure de $M$. pruriens s'élève à un montant de 786060 FCFA. Ce résultat est proche de la marge brute de $792600 \mathrm{FCFA} / \mathrm{ha}$ obtenue au Burkina Faso (Coulibaly et al., 2017). Pour le cas de la culture de M. pruriens associé au maïs dans l'ensemble des sites d'étude, la marge brute a été de $850917 \mathrm{FCFA} / \mathrm{ha}$. Ce résultat est nettement supérieur à une marge brute de 200773 FCFA/ha obtenue dans l'association du maïs au $M$. pruriens au Burkina Faso (Coulibaly et al., 2012). Cette grande différence se situe au niveau des prix moyens affectés aux produits bruts. Ces auteurs n'ont pas aussi pris en compte les frais de la récolte.

\section{Conclusion}

Les résultats de cette étude montrent que la culture pure de $M$. pruriens ou son association au maïs est performante dans la production du fourrage pour les animaux. Dans l'association, la production est plus élevée qu'en culture pure. La culture est également rentable économiquement pour les agroéleveurs. Dans les conditions climatiques de la zone d'étude, la date optimale de semis se situe dans la deuxième décade du mois de juillet pour la culture pure de $M$. pruriens. Elle est plus indiquée dans la première décade du mois d'aout pour le cas de l'association avec le maïs tout en respectant l'écart entre les deux semis (25-30 jours). À partir de ces dates optimales, tout retard de semis entraîne une baisse de rendement des cultures.

Pour une meilleure insertion de la technologie au sein du système de production agricole des exploitants agricoles de la zone, il serait nécessaire de mettre en place des dispositifs d'accompagnement des systèmes de production et d'utilisation de $M$. pruriens (semences, fourrages) et leur commercialisation (toute une chaîne de valeur).

\section{CONFLITS D'INTERÊTS}

Les auteurs déclarent qu'il n'existe aucun conflit d'intérêts.

\section{CONTRIBUTIONS DES AUTEURS}

Le co-auteur DC a participé à la conception et la planification de l'étude. Les co-auteurs $\mathrm{AB}, \mathrm{AKK}$ et NFD ont participé à l'analyse statistique, l'interprétation des données et à la rédaction de la première version du manuscrit. Quant à $\mathrm{BK}$, il a apporté son expertise dans l'analyse économique.

\section{REMERCIEMENTS}

Les auteurs remercient le personnel de la Délégation du programme Bovin du Centre Régional de Recherche Agronomique (CRRA) de Sikasso. Ils remercient également le projet PASE II (projet d'Appui à l'Amélioration de la Gouvernance de la filière coton dans sa nouvelle configuration institutionnelle et à la productivité et à la durabilité des Systèmes d'Exploitation en zone cotonnière) pour le financement des activités de terrain et les producteurs collaborateurs des sites d'études 
pour leur dévouement dans la conduite des essais.

\section{REFERENCES}

Ba A, Lesnoff M, Moulin CH. 2011. Demographic dynamics and off-take of cattle herds in south Mali. Trop. Anim. Health Prod., (43): 1101-1109. DOI : 10.1007/s11250-011-9808-2

Blanchard M. 2010. Gestion de la fertilité des sols et rôle du troupeau dans les systèmes coton-céréales-élevage au Mali-Sud: savoirs techniques locaux et pratiques d'intégration agriculture élevage. Océan, Atmosphère. Thèse de doctorat, Université Paris-Est, France, p. 285.

Boudet G, Rivière R. 1968. Emploi pratique des analyses fourragères pour l'appréciation des pâturages tropicaux. Rev. Elev. Médecine Vét. Pays Trop., 21(2): 227-266. DOI : https://agritrop.cirad.fr/444495/

Carsky RJ, Douthwaite B, Manyong VM, Sanginga N, Schulz S, Vanlauwe B, Diels J, Keatinge JDH. 2003. Amélioration de la gestion des sols par l'introduction de légumineuses dans les systèmes céréaliers des savanes africaines. Cah. Agric. 12(4) : 227-233.

Coulibaly D. 2008. Changements sociotechniques dans les systèmes de production laitière et commercialisation du lait en zone péri-urbaine de Sikasso, Mali. Thèse de doctorat. Paris, Agro Paris Tech, p. 399.

Coulibaly D, Poccard CR, Ba A. 2009. Dynamiques territoriales et changements des modes de gestion des ressources pastorales au Mali Sud (Mali). Renc. Rech. Ruminants, 16:357-360. DOI : https://agritrop.cirad.fr/552944/

Coulibaly D, Ba A, Dembélé B, Sissoko, F. 2017. Développement des systèmes de production innovants d'association mais/légumineuses dans la zone subhumide du Mali. Agronomie Africaine SP., 29(1): $\quad 1-10 . \quad$ DOI : https://www.ajol.info/index.php/aga/artic le/view/163158
Coulibaly K, Vall E, Autfray P, Sedogo MP 2012. Performance technico-économique des associations maïs/niébé et maïs/mucuna en situation réelle de culture au Burkina Faso: potentiels et contraintes. Tropicultura, 30(3): 147154.

DOI : http://www.tropicultura.org/text/v28n3/1 33.pdf

Coulibaly K, Alain PK, Gomgnimbou K, Mamadou T, Nacro H, Sedogo M. 2017. Effets des associations maïslégumineuses sur le rendement du maïs (Zea mays L.) et la fertilité d'un sol ferrugineux tropical à l'Ouest du Burkina Faso. Afrique SCIENCE, 13(6): 226-235. https://www.researchgate.net/publication /321621867

Diatta A, Dieng A, Diaw M.T, Nesseim T.D.T, Ndiaye S. 2020. Production et valeur nutritive de fourrages de pois Mascate (Mucuna pruriens (L.) DC. var. utilis (Wall. ex Wight) Baker ex Burck) cv. Ghana cultivé dans le Centre et Nord bassin arachidier sénégalais. Int. J. Biol. Chem. Sci., 14(4) : 1262-1272. DOI : 10.4314/ijbcs.v14i4.8

Djenontin AJP, Houinato M, Toutain B, Sinsin B. 2009. Pratiques et stratégies des éleveurs face à la réduction de l'offre fourragère au Nord-Est du Bénin. Sci. Chang. Planétaires Sécher, 20(4): 346353. DOI : $10.1684 / \mathrm{sec} .2009 .0204$

Haro H, Semde K, Bahadio K, Sanon, K.B. 2020. Effet de l'inoculation mycorhizienne avec des souches des champignons mycorhiziens arbusculaires sur la croissance de Mucuna pruriens (L.) DC en condition contrôlée. Int. J. Biol. Chem. Sci., 14(3) : 1065-1073. DOI : https://doi.org/10.4314/ijbcs.v14i3.32

Hauser S, Nolte C. 2002. Biomass production and $\mathrm{N}$ fixation of five Mucuna pruriens varieties and their effect on maize yields in the forest zone of Cameroon. J. Plant Nutr. Soil Sci., 165(1) : 101-109.

INSTAT. 2016. Rapport Annuaire Statistique du Mali (Bamako: Institut National de la Statistique, p.76. http://www.instatmali.org/contenu/pub/anuair16_pub.pdf 
Kiema A, Sawadogo I, Ouedraogo T, Nianogo, AJ. 2012. Stratégies d'exploitation du fourrage par les éleveurs de la zone sahélienne du Burkina Faso. Int. J. Biol. Chem. Sci., 6(4) : 1492-1505. DOI : http://dx.doi.org/10.4314/ijbcs.v6i4.8

Klein HD, Rippstein G, Huguenin J, Toutain B, Guerin H, Louppe D. 2014. Les cultures fourragères. CTA et Presses Agronomiques de Gembloux, Collection Agricultures tropicales en poche: Quae.

Koutou M, Coulibaly K, Vall E, Bognini S, Pooda RKW, Sanogo L, Chi E. 2009. Evaluation d'impact et taux d'adoption d'une innovation agropastorale: cas de l'introduction du Mucuna deeringiana, légumineuse à double fin (fertilité, fourrage). In : Evaluation des impacts des innovations dans les systèmes de production et les territoires agropastoraux d'Afrique de l'Ouest: quelles méthodes, quels indicateurs? : Actes de l'atelier, 14 décembre 2009, Ouagadougou, Burkina Faso, p. 16. https://agritrop.cirad.fr/559200/

Koutou M, Havard M, Ouedraogo D, Sangaré M, Toillier A, Thombiano T, Vodouhé SD. 2016. Facteurs d'adoption des innovations d'intégration agricultureélevage : cas du Mucuna pruriens en zone cotonnière ouest du Burkina Faso. Tropicultura, 34(4): 424-439. DOI : http://www.tropicultura.org/eng/content/ v34n4.html.

Pamba M, Muwo J.C, Mwengi I. 2018. Etude des possibilités de production de maïs (Zea mays L.) et de soja (Glycine max (L.) Merr.) Sur couverture de Mucuna pruriens (L.) DC. dans les conditions écologiques de Kikwit en République Démocratique du Congo. Revue Africaine d'Environnement et d'Agriculture, 1(1) : 30-35.

Sanogo OM, Ridder N, Keulen HV. 2010. Diversité et dynamique des exploitations agricoles mixtes agriculture-élevage au sud du Mali. Cahiers. Agricultures, 19(3): 185-193. DOI : 10.1684/agr.2010.0401

Sissoko F, Diarra S, Traore, M. 2020. Le semis direct sous couverture végétale: une opportunité de mise en place rapide du cotonnier en culture pluviale au Mali. Int. J. Biol. Chem. Sci., 14(3): 722-738. DOI: https://doi.org/10.4314/ijbcs.v14i3.7

Soumaré M, Bazile D, Diallo K, Vaksmann M, Kouressy M. 2008. Diversité agroécosytémique et devenir des céréales traditionnelles au sud du Mali. Cahiers. Agricultures, 17(2): 79-85. DOI : 10.1684/agr.2008.0184. 